\title{
Parameter Estimation of the ALBA Autonomous Surface Craft
}

\author{
Melanie M. Valdivia-Fernandez ${ }^{1}$, Brayan A. Monroy-Ochoa ${ }^{2}$, Daniel D. Yanyachi ${ }^{3}$, Juan C. Cutipa-Luque ${ }^{4}$ \\ Electronic Engineering Professional School ${ }^{1,2,3}$ \\ Universidad Nacional de San Agustín de Arequipa, Av. Venezuela s/n, Cercado, Arequipa, Peru \\ Pedro Paulet's Astronomic and Aerospace Institute ${ }^{4}$ \\ Universidad Nacional de San Agustín de Arequipa, Cerro San Francisco s/n, Characato, Arequipa, Peru
}

\begin{abstract}
Arequipa region holds the largest extension of the Peruvian littoral at the Pacific sea, has also fresh water resources composed of rivers and lagoons from the coast to the Andes highland. The ALBA vehicle is a low-cost autonomous surface vessel with open source architecture that is being developed to support water monitoring tasks in the region. This article deals with the nonlinear identification problem for an autonomous surface craft and the maximum likelihood estimation approach is used to estimate its parameters. The parametric nonlinear model is considered with simulated and experimental data. The results shows good fitting values when two, three and a maximum four parameters are estimated.
\end{abstract}

Keywords-Autonomous surface craft; parameter estimation; modeling; maximum likelihood; nonlinear; zigzag

\section{INTRODUCTION}

Water, the most precious resource for human being, is being vulnerable to contamination at present since there is enough evidence [1]. The Mar de Grau is the Peruvian sea with abundant marine species, presents a littoral of 3079.50 $\mathrm{km}$ and a breadth extension of $370.4 \mathrm{~km}(200 \mathrm{nmi})$. Arequipa is the south region of Peru with the largest littoral, more than $500 \mathrm{~km}$. There is an open area for a sustainable exploration and monitoring these resources and the fresh waters that feed them.

The monitoring of sea conditions is commonly carried out using manned vessels, following standard international procedures and agreements. However, these large vessels cannot work in coastal areas and estuary locals due to the risk of crashing with rocks, irregularities in seabed, and currents. The use of autonomous surface crafts (ASCs) is an alternative and has advantages, such as low dimensions, zero human risk, able to explore shallow waters.

The main contribution of this paper is to estimate a greater number of parameters for the ALBA ASC, using the maximum likelihood approach with simulated and actual data, the method used in this paper is called maximum likelihood estimation (MLE) and allows us to identify many parameters at a time, therefore MLE is used for large samples and is very versatile and accurate because it works estimating not only from the values obtained of the inertial sensor and of the position sensor so it is reliable and even being able to have initial conditions. The organization of this document is as follows: Section 1 provides topics on the importance of water and its exploration using ASCs. For this, the identification of parameters and the importance of using the MLE method is done; Section 2 presents the work done on parameter estimation and ASCs; Section 3 presents the mathematical model of the ALBA ASC in nonlinear representation; Section 4 presents the maximum likelihood parameter estimation approach to identify the ALBA ASC; Section 5 presents the experimental tests and their achieved estimated parameters; Section 6 provides the conclusion.

\section{BACKGROUND}

The ASCs are executing different missions around the world and their developments involve multidisciplinary areas, such as modeling, identification, navigation, control, guidance, path planning, etc. The Charlie ASC, for instance, carries out surface micro layer sampling with its real-time platform composed of navigation, guidance and control [2]. Another ASC, powered with solar energy, has navigation, guidance and collision avoidance systems to accomplish missions of water quality and greenhouse gas emissions measurements in lakes. [3].

In [4], the authors describe the modeling and identification of an ASC in a wide range of speeds and glide conditions, obtaining good estimated parameters that have been used in the proportional-derivative (PD) controller synthesis. There are other approaches to estimate nonlinear model parameter, such as the symbolic regression using genetic programming [5]. The model parameter can also be identified using experimental towing tank and open water self-propelled tests, as in [6]. The nonlinear parameters estimation is presented in [7] using the maximum likelihood approach and applied to autonomous underwater vehicle in [8]. This method maximizes the likelihood function of innovation variables, which is the difference between the output measured variable and the output estimated variable. In [9], the authors present the recursive least squares optimization approach to determine the linear and nonlinear parameters of an autonomous underwater vehicle (AUV). In [10], the authors present the parametric identification model of a ship based on the least squares approach, validating by means of high precision of identified hydrodynamic derivatives. Identifiability property can be verified before to apply a parameter estimation approach and there are some linear algebra tools that can solve this problem indeed when the model to be identified is nonlinear. These tools can be found in [11], [12], [13], [14]. 


\section{ALBA ASC MODELING}

ALBA is a low-cost ASC, developed on an inflatable boat, used for water quality monitoring and scientific study. This vehicle has a trolling motor and a servomechanism that changes the force direction for maneuvering. The vehicle has a control architecture composed of inertial navigation sensors, wireless communication, and microcontrollers to execute navigation, guidance and control algorithms. Fig. 1 shows the picture of the cited vehicle under Lake tests, a detailed description of its development can be found in [15]. Fig. 2 presents a diagram of the servomechanism structure developed for turning the trolling motor, with $0.2462 \mathrm{~m}$ height and $0.511 \mathrm{~m}$ long. The servomotor is located on the right side and covers a space of $0.062 \times 0.094 \times 0.029 \mathrm{~m}$. Fig. 2 (b) shows the clamps where the trolling helm is installed.

The ALBA model is expressed according to standard notation used in maritime vehicles [16]. The dynamics of 6 degrees-of-freedom (DOF) are represented with two coordinate systems, one named earth-fixed $\eta=(x, y, z, \phi, \theta, \psi)$ and another named body-fixed $\nu=(u, v, w, p, q, r)$ (Fig. 3). The dynamic equation is given by (1) and the kinematic transformation between earth-fixed and body-fixed frames is expressed by (2), respectively:

$$
\begin{aligned}
M \dot{\nu}+C(\nu) \nu+D(\nu) \nu+g(\eta) & =\tau, \\
\dot{\eta} & =J(\eta) \nu,
\end{aligned}
$$

where $J(\eta)$ is the coordinate transformations; $M$ is the mass matrix composed of rigid body mass and added mass; $C(\nu)$ includes terms of centripetal, Coriolis and rigid body; $D(\nu)$ is the damping matrix and $\tau$ is the control effort vector. Table I presents the main features of the ALBA ASC.

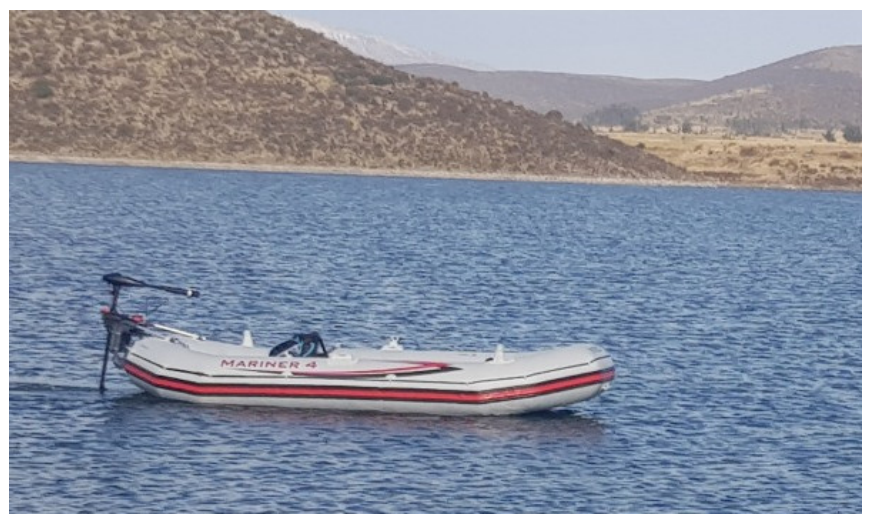

Fig. 1. ALBA ASC Operating in a Lake [15].

For the ALBA ASC, it is reduced in dynamics to 2-DOF that correspond to the body $\eta=(v, r)^{T}$ and inertial $\nu=$ $(y, \psi)^{T}$ frames, respectively. The hydrostatic forces in these two directions are null and the strip theory is used to obtain theoretical vehicle parameters [17]. The nonlinear model is given for very small values of the propeller angle $\delta_{p}$ and surge velocity constant $u_{0}=$ cte.

$$
\begin{array}{r}
\left(m-Y_{\dot{v}}\right) \dot{v}+\left(m x_{G}-Y_{\dot{r}}\right) \dot{r}=Y_{v} u_{0} v+Y_{r} u_{0} r+ \\
Y_{v|v|} v|v|+Y_{r|r|} r|r|-m u_{0} r+b+k_{u_{0}} \sin \delta_{p} \\
\left(m x_{G}-N_{\dot{v}}\right) \dot{v}+\left(I_{z}-N_{\dot{r}}\right) \dot{r}=N_{v} u_{0} v+N_{r} u_{0} r+ \\
N_{v|v|} v|v|+N_{r|r|} r|r|-m x_{G} u_{0} r-l_{x_{G}} k_{u_{0}} \sin \delta_{p}
\end{array}
$$
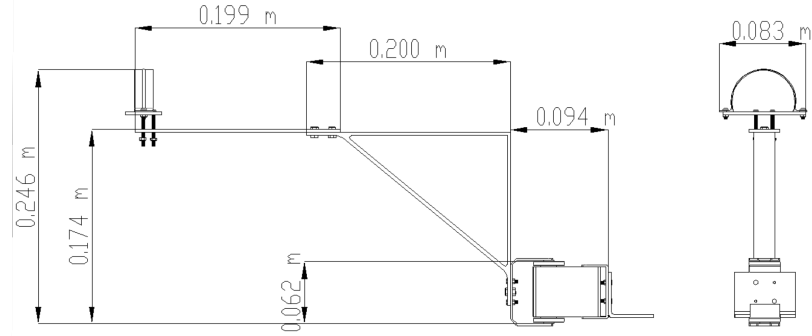

a) Side View

b) Front View

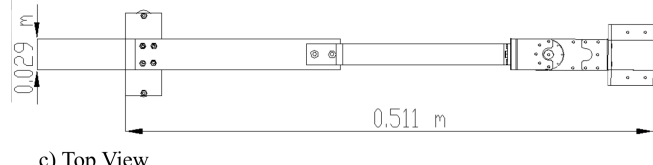

Fig. 2. Turning Propeller Mechanism.

Earth-frame

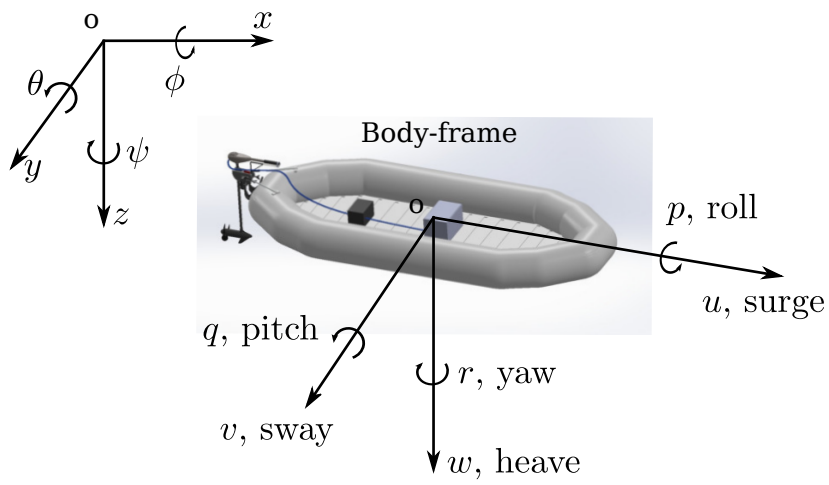

Fig. 3. ALBA ASC Coordinate System.

$$
\begin{gathered}
\dot{y}=u_{0} \sin (\psi)+v \cos (\psi), \\
\dot{\psi}=r,
\end{gathered}
$$

where $k_{u_{0}}=99$ is the propeller coefficient, $l_{x_{G}}=1.79 \mathrm{~m}$ is the distance from the propeller location to the gravity center, and the rest hydrodynamic derivatives are described in Table II. The propeller angle in this vehicle is limited by $\pm 25^{\circ}$ degrees considered sufficient to generate effort to maneuver the whole craft.

Using straightforward transformation, the nonlinear model of the ALBA ASC (3)-(6) can be expressed in the standard

TABLE I. ALBA ASC MAIN FEATURES

\begin{tabular}{l|l}
\hline Features & Value \\
\hline \hline Length $(L)$ & $3.14 \mathrm{~m}$ \\
\hline Width & $1.45 \mathrm{~m}$ \\
\hline Gravity center $\left(x_{G}\right)$ & $0.15 \mathrm{~m}$ \\
\hline Propeller location $\left(l_{x_{G}}\right)$ & $1.79 \mathrm{~m}$ \\
\hline Mass $(m)$ & $125 \mathrm{~kg}$ \\
\hline Inertia around $\mathrm{z}$ axis $\left(I_{z}\right)$ & 123.84 \\
\hline Cruise speed $\left(u_{0}\right)$ & $1 \mathrm{~m} / \mathrm{s}$ \\
\hline Maximum speed & $4 \mathrm{~m} / \mathrm{s}$ \\
\hline Autonomy & $3 \mathrm{hours}$ \\
\hline
\end{tabular}


TABLE II. ALBA HYDRODYNAMIC PARAMETERS

\begin{tabular}{l|l|l|l}
\hline Parameters & Value & Unit & Description \\
\hline \hline$Y_{\dot{v}}$ & -571.0619 & $\mathrm{~kg}$ & Added mass \\
\hline$Y_{\dot{r}}$ & -101.9671 & $\mathrm{~kg} . \mathrm{m} / \mathrm{rad}$ & Added mass \\
\hline$N_{\dot{v}}$ & -101.9671 & $\mathrm{k} . \mathrm{m}$ & Added mass \\
\hline$N_{\dot{r}}$ & -232.9491 & $\mathrm{~kg} \cdot \mathrm{m}^{2} / \mathrm{rad}$ & Added mass \\
\hline$Y_{v}$ & -139 & $\mathrm{~kg} / \mathrm{s}$ & Linear drag \\
\hline$Y_{v|v|}$ & -854.7687 & $\mathrm{~kg} / \mathrm{m}$ & Nonlinear drag \\
\hline$Y_{r}$ & -43.8331 & $\mathrm{~kg} \cdot \mathrm{m}^{2} /{\mathrm{rad} . \mathrm{s}^{2}}$ & Linear cross drag \\
\hline$Y_{r|r|}$ & -228.8418 & $\mathrm{~kg} \cdot \mathrm{m} / \mathrm{rad}^{2}$ & Nonlinear cross drag \\
\hline$N_{v}$ & 70.5207 & $\mathrm{~kg} . \mathrm{m} / \mathrm{s}$ & Linear cross drag \\
\hline$N_{v|v|}$ & -143.9553 & $\mathrm{~kg}$ & Nonlinear cross drag \\
\hline$N_{r}$ & -101.9671 & $\mathrm{~kg} . \mathrm{m}^{2} /{\mathrm{rad} . \mathrm{s}^{2}}^{2}$ & Linear drag \\
\hline$N_{r|r|}$ & -884.0436 & $\mathrm{~kg} \cdot \mathrm{m}^{2} / \mathrm{rad}^{2}$ & Nonlinear drag \\
\hline$b$ & -10 & $\mathrm{~kg}$ & Offset in sway \\
\hline
\end{tabular}

form:

$$
\begin{aligned}
& \dot{\mathrm{x}}=\mathrm{f}(\mathrm{x}, \mathrm{u}), \\
& \mathrm{y}=\mathrm{g}(\mathrm{x}, \mathrm{u}),
\end{aligned}
$$

where $\mathbf{x}=\left[\begin{array}{llll}v & r & y & \psi\end{array}\right]^{T}$ is the state space vector, $\mathbf{y}=$ $\left[\begin{array}{lll}r & y & \psi\end{array}\right]^{T}$ is the output vector, $\mathrm{u}=\delta_{p}$ is the control input, and $f$ and $g$ are nonlinear functions. More details of nonlinear and linearizing models can be found in [15].

\section{Parameter Estimation Approach}

The parameters estimation approach is presented here based on an optimization problem. The goal is to maximize a likelihood cost function, which means an expression of the output error or difference between measurement and output variables (Fig. 4). This is a nonlinear approach because the model to be identified presents nonlinearities (7). There should be included the parameter vector $\Theta$, the discrete time measurement variable $\mathbf{z}(k)$ with $N$ samples, w state noise variable with its distribution matrix $G$, and v measurement noise variable with distribution matrix $F$. Then, the nonlinear model to be used in MLE approach is rewritten as:

$$
\begin{aligned}
\dot{\mathrm{x}} & =\mathrm{f}(\mathrm{x}, \mathrm{u}, \Theta)+\mathrm{G}(\mathrm{w}) \\
\mathrm{y} & =\mathrm{g}(\mathrm{x}, \mathrm{u}, \Theta) \\
\mathrm{z}(k) & =\mathrm{y}(k)+\mathrm{Fv}(k)
\end{aligned}
$$

The prediction error is given by:

$$
\mathrm{q}(k)=[\hat{\mathrm{y}}(k)-\mathrm{y}(k)]
$$

Then, a likelihood function is expressed in function of $q$ and its respective covariance matrix $\mathcal{B}$ :

$$
\begin{aligned}
p(\mathrm{y} \mid \Theta)= & (2 \pi)^{-m / 2}|\mathcal{B}|^{-n / 2} . \\
& \exp \left[-\frac{1}{2} \sum_{k=1}^{n}[\mathbf{q}(k, \Theta)]^{T} \mathcal{B}^{-1}[\mathbf{q}(k, \Theta)]\right]
\end{aligned}
$$

where $n$ is the dimension of state space vector $\mathrm{x}$ and $m$ is the dimension of the measurement vector $y$. The value of $\Theta$ is estimated through the maximization of this likelihood function, as follows:

$$
\hat{\Theta}=\underset{\Theta}{\arg \max } p(\mathrm{y} \mid \Theta)
$$

The likelihood expression (10) can be transformed using the relation $-\ell n(p(\mathrm{y} \mid \Theta))$, and neglected the constant term [18], [7]:

$$
J(\Theta)=\frac{1}{2} \sum_{i=1}^{N}\left\{[\mathrm{q}(k, \Theta)]^{T} \mathcal{B}^{-1}[\mathrm{q}(k, \Theta)]+\ln |\mathcal{B}|\right\}
$$

Therefore, minimize the functional $J(\Theta)$ is equivalent to maximize the likelihood function with a great advantageous for computational purposes. There are many types of algorithms that solve the problem of the optimization, such as GaussNewton (GN) and Levenberg-Marquardt (LM).

To achieve an optimal $\Theta$, the cost function $J(\Theta)$ should be approximated to a parabolic function using the well known Taylor series [7]:

$$
\begin{aligned}
J\left(\Theta_{0}+\Delta \Theta\right) \cong & J\left(\Theta_{0}\right)+\left.\Delta \Theta^{T} \frac{\partial J}{\partial \Theta}\right|_{\Theta=\Theta_{0}} \\
& +\left.\frac{1}{2} \Delta \Theta^{T} \frac{\partial^{2} J}{\partial \Theta \partial \Theta^{T}}\right|_{\Theta=\Theta_{0},} \Delta \Theta
\end{aligned}
$$

where $\Theta_{0}$ is the nominal vector parameter. The optimization is obtained under the constraint:

$$
\frac{\partial}{\partial \Theta}\left[J\left(\Theta_{0}+\Delta \Theta\right)\right]=0
$$

Solving expression (13) with the constraint given in (14), the variation of the estimated parameter vector $\Delta \hat{\Theta}$ is:

$$
\Delta \hat{\Theta}=-\left.\left[\left.\frac{\partial^{2} J}{\partial \Theta \partial \Theta^{T}}\right|_{\Theta=\Theta_{0}}\right]^{-1} \frac{\partial J}{\partial \Theta}\right|_{\Theta=\Theta_{0}}
$$

Let the Hessian matrix be a non-singular matrix, the estimated parameter vector can be expressed as follows:

$$
\hat{\Theta}=\Theta_{0}+\Delta \hat{\Theta}
$$

Considering the approximation (13) for the cost function, in the next iteration, the process will be repeated assuming the estimated vector as a nominal parameter $\Theta_{0}=\hat{\Theta}$. Therefore, the generalized recursive equation is expressed as:

$$
\Theta_{j+1}=\Theta_{j}-\left[\nabla_{\Theta}^{2} J\left(\Theta_{j}\right)\right]^{-1} \nabla_{\Theta}^{T} J\left(\Theta_{j}\right)
$$

where $\nabla$ is the gradient of $J$ whose Hessian matrix is $\nabla_{\Theta}^{2} J\left(\Theta_{j}\right)$. Compute of Hessian matrix demands a huge computational effort which can be avoided by the GN algorithm:

$$
\nabla_{\Theta}^{2} J(\Theta)=\sum_{k=1}^{n}\left[\nabla_{\Theta} \hat{\mathrm{y}}_{k}(\Theta)\right]^{T}[\mathcal{B}]^{-1}\left[\nabla_{\Theta} \hat{\mathrm{y}}_{k}(\Theta)\right]
$$

where the terms of the second order derivatives are removed. The gradient of the estimated output, $\nabla_{\Theta} \hat{\mathrm{y}}_{k}(\Theta)$, is named sensitivity function.

The LM algorithm is an extension of the GN, whose principal idea consists in modify $\nabla_{\Theta}^{2} J(\Theta)$ by the expression $\nabla_{\Theta}^{2} J(\Theta)+\lambda I$ in the Eq. (17). The inversion of the matrix is not yielded in explicit manner and, now, it will be solved 
by singular value decomposition (SVD) according to the expression:

$$
\left[\nabla_{\Theta}^{2} J(\Theta)+\lambda \mathbf{I}\right] \Delta \hat{\Theta}=\nabla_{\Theta}^{T} J\left(\Theta_{j}\right)
$$

The above LM algorithm [19] solves the problem of singularity in the Hessian matrix. Additionally, the LM algorithm works like a GN algorithm for small values of $\lambda$. These optimization algorithms are already present in libraries of noncommercial and commercial softwares, such as Gnu-Octave and Matlab.

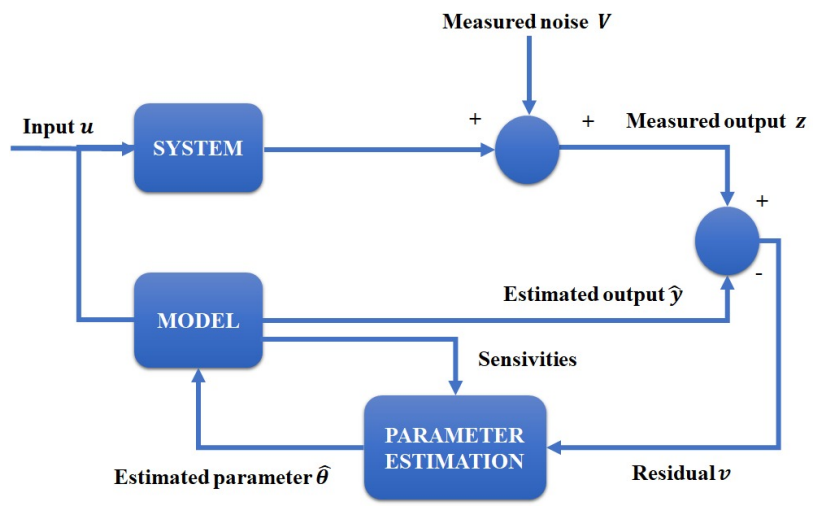

Fig. 4. Parameter Estimation via Output Error.

\section{RESUlTS}

This section gives the results of the approach applied to the ALBA ASC. For data generated in simulator and data obtained experimentally, the parameter vector defined in (8) is expressed relative to the nonlinear model as:

$$
\Theta=\left[\begin{array}{llll}
\Theta_{1} & \Theta_{2} & \Theta_{3} & \Theta_{4}
\end{array}\right]^{T},
$$

where $\Theta_{1}=Y_{\dot{v}}, \Theta_{2}=I_{z}, \Theta_{3}=N_{v}$, and $\Theta_{4}=b$. Three cases are analyzed in order to estimate a greater number of parameters.

\section{A. Simulated}

The nonlinear model of the ALBA ASC expressed by (3)-(6) is implemented in Matlab/Simulink as shown in Fig. 5. The upper block named ALBA USV (unmanned surface vehicle) presents the nonlinear dynamics of the vehicle, and the lower block named Zig-zag maneuvering presents the zigzag maneuver generated numerically.

Fig. 6 presents the plot of data generated using this software resource, where $\psi$ is the yaw angle in zigzag course due to the switching control of the propeller angle $\delta_{p}$ between $\pm 20^{\circ}= \pm 0.3490 \mathrm{rad}$. This switching control signal is in closed loop and is activated by the yaw angle limits given also between $\pm 20^{\circ}= \pm 0.3490 \mathrm{rad}$. The yaw rate angle $r$ follows an oscillatory behavior indicating the necessary angular rate of the vehicle to approach this zigzag maneuver, the initial condition for this numerical test is $v=0.001 \mathrm{~m} / \mathrm{s}$, $r=0.001 \mathrm{rad} / \mathrm{s}, y=0.001 \mathrm{~m}$ and $\psi=-30 \times \mathrm{pi} / 180 \mathrm{rad}$

The simulated data were obtained with different hydrodynamic parameters moved purposefully to $25 \%$ respect to the theoretical values (nominal values) given in Table II.

Table III presents the case where two parameters are estimated using the MLE algorithm. The fitting between identified model response and simulated data is $94.56 \%$ for the yaw rate $r, 57.71 \%$ for the $y$ position, and $58.88 \%$ for the yaw angle $\psi$ (Table IX). Table IV presents the case where three parameters are estimated using the MLE algorithm. The fitting between identified model response and simulated data is $94.55 \%$ for the yaw rate $r, 56.77 \%$ for the $y$ position, and $58.98 \%$ for the yaw angle $\psi$. (Table IX). Table V presents the case where four parameters are estimated using the MLE algorithm. The fitting between identified model response and simulated data is $90.49 \%$ for the yaw rate $r, 71.38 \%$ for the $y$ position, and $83.16 \%$ for the yaw angle $\psi$ (Table IX). Fig. 7 presents this last comparison validating the approach used here.

TABLE III. ESTIMATION WITH 2 PARAMETERS.

\begin{tabular}{l|r|r|l}
\hline \hline Parameter & Estimated value & Nominal value & Maneuver \\
\hline$\Theta_{3}$ & 102.551309 & 70.520700 & $\pm 20(\pi / 180) \mathrm{rad}$ \\
$\Theta_{4}$ & -8.139283 & -10.00000 & $\pm 20(\pi / 180) \mathrm{rad}$ \\
\hline \hline
\end{tabular}

TABLE IV. ESTIMATION WITH 3 PARAMETERS.

\begin{tabular}{l|r|r|l}
\hline \hline Parameter & Estimated value & Nominal value & Maneuver \\
\hline$\Theta_{2}$ & 172.83619 & 123.844500 & $\pm 20(\pi / 180) \mathrm{rad}$ \\
$\Theta_{3}$ & 102.722541 & 70.520700 & $\pm 20(\pi / 180) \mathrm{rad}$ \\
$\Theta_{4}$ & -8.3401932 & -10.00000 & $\pm 20(\pi / 180) \mathrm{rad}$ \\
\hline \hline
\end{tabular}

TABLE V. ESTIMATION WITH 4 PARAMETERS.

\begin{tabular}{l|r|r|l}
\hline \hline Parameter & Estimated value & Nominal value & Maneuver \\
\hline$\Theta_{1}$ & -897.157150 & -571.061900 & $\pm 20(\pi / 180) \mathrm{rad}$ \\
$\Theta_{2}$ & 188.315315 & 123.844500 & $\pm 20(\pi / 180) \mathrm{rad}$ \\
$\Theta_{3}$ & 103.501259 & 70.520700 & $\pm 20(\pi / 180) \mathrm{rad}$ \\
$\Theta_{4}$ & -8.923712 & -10.00000 & $\pm 20(\pi / 180) \mathrm{rad}$ \\
\hline \hline
\end{tabular}

\section{B. Experimental}

The experimental tests were carried out in the Tingo lagoon in Arequipa. The inertial navigation system provided the yaw rate $r$ and yaw angle $\psi$ at sampling time of $0.1 \mathrm{~s}$, a global positioning system (GPS) provided the position $y$ at sampling time of $1 \mathrm{~s}$. There was a need to use oversampling technique from $1 \mathrm{~s}$ to $0.1 \mathrm{~s}$ for the $y$ data in order to feed the estimation algorithm used here. The control $\delta_{p}$ signal is also provided at $0.1 \mathrm{~s}$ sampling time from the embedded electronic of ALBA ASC, described in [15].

Fig. 8 presents the plot of experimental data, where $\psi$ is the yaw angle in zigzag course due to the switching control of the propeller angle $\delta_{p}$ between $\pm 20^{\circ}= \pm 0.3490 \mathrm{rad}$. This switching control signal is in closed loop and is activated by the yaw angle limits given also between $\pm 20^{\circ}= \pm 0.3490$ $\mathrm{rad}$, the initial condition for this numerical test is $v=0 \mathrm{~m} / \mathrm{s}$, $r=0 \mathrm{rad} / \mathrm{s}, y=0.01 \mathrm{~m}$ and $\psi=-30 \times \mathrm{pi} / 180 \mathrm{rad}$ 
. The yaw rate angle $r$ follows an oscillatory behavior indicating the necessary angular rate of the vehicle to approach this zigzag maneuver.

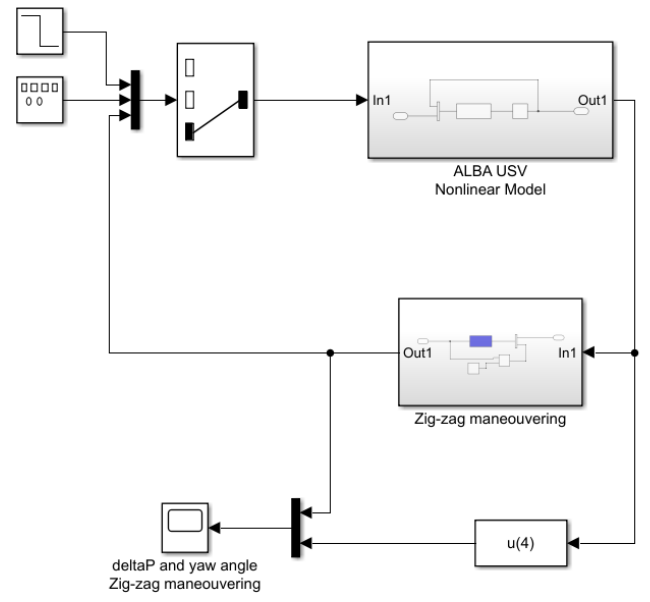

Fig. 5. Nonlinear Model Simulink.

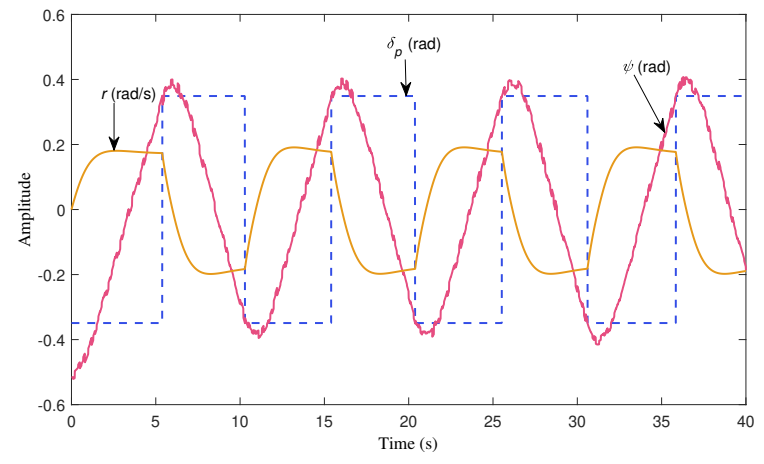

Fig. 6. Simulated Zigzag Maneuver.

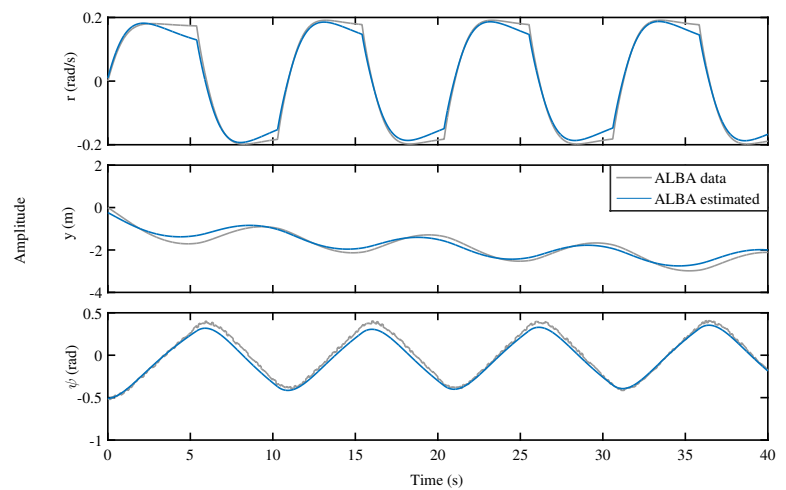

Fig. 7. Comparison Responses between the Identified Model and the Simulated Data.
Table VI presents the case where two parameters are estimated using the MLE algorithm. The fitting between identified model response and experimental data is $76.05 \%$ for the yaw rate $r, 16.27 \%$ for the $y$ position, and $63.35 \%$ for the yaw angle $\psi$ (Table IX). Table VII presents the case where three parameters are estimated using the MLE algorithm. The fitting between identified model response and experimental data is $75.78 \%$ for the yaw rate $r, 15.15 \%$ for the $y$ position, and $63.45 \%$ for the yaw angle $\psi$ (Table IX). Table VIII presents the case where four parameters are estimated using the MLE algorithm. The fitting between identified model response and simulated data is $75.71 \%$ for the yaw rate $r$, $15.31 \%$ for the $y$ position, and $64.46 \%$ for the yaw angle $\psi$ (Table IX). Fig. 9 presents this comparison validating the approach used here.

Table IX summarizes the numerical and experimental results carried out to estimate the main parameters of the ALBA. The fitting between the identified model and experimental data are good and above to 50\%, except for the $y$ position. As noted in model structure (3)-(6), its differential equation does not exert significant contribution in the whole model. Moreover, in autonomous vehicles [16], the $y$ kinematic commonly compromises the observability and controllability linear properties. Here, there is an unsolved and open area for autonomous surface craft consisting in to examine identifiability properties and advances recently developed for biologic systems [13], [14].

\section{CONCLUSIONS}

A nonlinear model for the ALBA autonomous surface vehicle was identified using the maximum likelihood estimation approach. This approach was initially tested numerically with data obtained through the vehicle dynamic simulator. The approach was then applied to the vehicle data obtained in experimental test maneuvers. The four estimated parameters compose the identified system for the ALBA, a low-cost vehicle destined to monitor water conditions of lagoons and shallow water of the Pacific sea. The fitting between identified model responses and data is quite good and above 50\%, guarantying the proposed approach and its application to this class of maritime vehicles. The fitting of $y$ position was not good and analysis using identifiability properties should be conducted further.

TABLE VI. ESTIMATION WITH 2 PARAMETERS.

\begin{tabular}{l|r|r|l}
\hline \hline Parameter & Estimated value & Nominal value & Maneuver \\
\hline$\Theta_{3}$ & 68.478772 & 70.520700 & $\pm 20(\pi / 180) \mathrm{rad}$ \\
$\Theta_{4}$ & -7.552532 & -10.000000 & $\pm 20(\pi / 180) \mathrm{rad}$ \\
\hline \hline
\end{tabular}

TABLE VII. ESTIMATION WITH 3 PARAMETERS.

\begin{tabular}{l|r|r|l}
\hline \hline Parameter & Estimated value & Nominal value & Maneuver \\
\hline$\Theta_{2}$ & 140.339921 & 123.844500 & $\pm 20(\pi / 180) \mathrm{rad}$ \\
$\Theta_{3}$ & 70.648283 & 70.520700 & $\pm 20(\pi / 180) \mathrm{rad}$ \\
$\Theta_{4}$ & -7.874004 & -10.000000 & $\pm 20(\pi / 180) \mathrm{rad}$ \\
\hline \hline
\end{tabular}


TABLE VIII. ESTIMATION WITH 4 PARAMETERS.

\begin{tabular}{l|r|r|l}
\hline \hline Parameter & Estimated value & Nominal value & Maneuver \\
\hline$\Theta_{1}$ & -819.501860 & -571.061900 & $\pm 20(\pi / 180) \mathrm{rad}$ \\
$\Theta_{2}$ & 124.193961 & 123.844500 & $\pm 20(\pi / 180) \mathrm{rad}$ \\
$\Theta_{3}$ & 73.561674 & 70.520700 & $\pm 20(\pi / 180) \mathrm{rad}$ \\
$\Theta_{4}$ & -7.160477 & -10.000000 & $\pm 20(\pi / 180) \mathrm{rad}$ \\
\hline \hline
\end{tabular}

TABLE IX. FITTING BETWEEN IDENTIFIED MODEL RESPONSE AND DATA (SIMULATED AND EXPERIMENTAL).

\begin{tabular}{c|c|c|c|c|c|c}
\hline \multirow{2}{*}{ Fitting } & \multicolumn{5}{|c}{ Number of estimated parameters } \\
\cline { 2 - 7 } & \multicolumn{3}{|c}{ Simulated } & \multicolumn{3}{c}{ Experimental } \\
\hline Response & two & three & four & two & three & four \\
\hline \hline$r$ & $94.56 \%$ & $94.55 \%$ & $90.49 \%$ & $76.05 \%$ & $75.78 \%$ & $75.71 \%$ \\
\hline$y$ & $57.71 \%$ & $56.77 \%$ & $71.38 \%$ & $16.27 \%$ & $15.15 \%$ & $15.31 \%$ \\
\hline$\psi$ & $58.88 \%$ & $58.98 \%$ & $83.16 \%$ & $63.35 \%$ & $63.45 \%$ & $64.46 \%$ \\
\hline
\end{tabular}

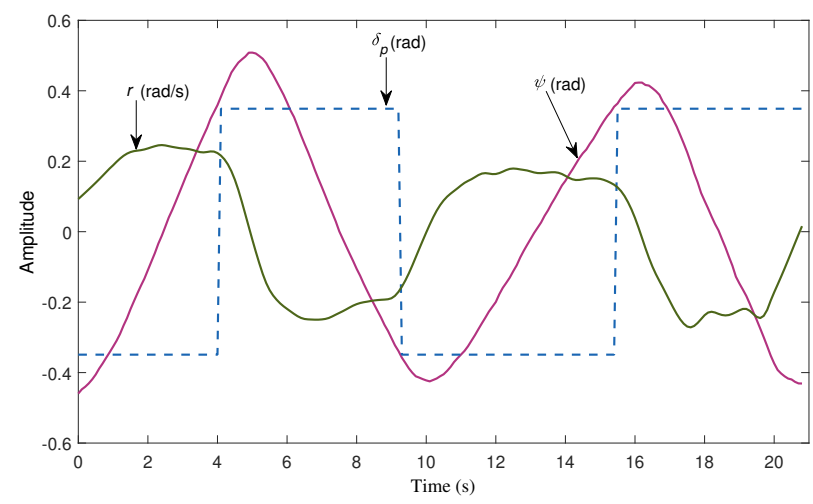

Fig. 8. Experimental Zigzag Maneuver.

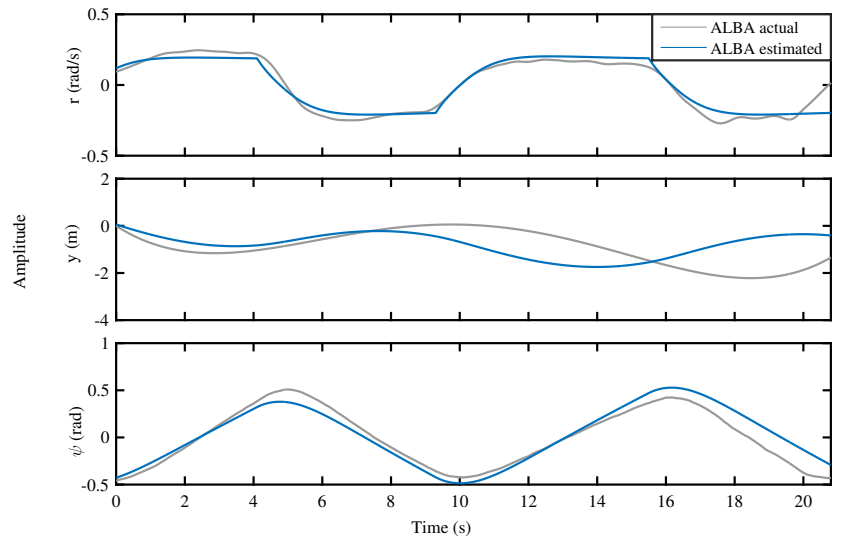

Fig. 9. Comparison Responses between the Identified Model and the Experimental Data.

\section{ACKNOWLEDGMENT}

The authors thank the Universidad Nacional de San Agustín de Arequipa for the incentives and financial support given to the construction of the ALBA vehicle, under grant number TP3-2018-UNSA.

\section{REFERENCES}

[1] V. Rajendran, S. Nirmaladevi D, B. Srinivasan, C. Rengaraj, and S. Mariyaselvam, "Quality assessment of pollution indicators in marine water at critical locations of the gulf of mannar biosphere reserve, tuticorin," Marine Pollution Bulletin, vol. 126, pp. $236-240,2018$. [Online]. Available: http://www.sciencedirect.com/science/article/pii/S0025326X17309372

[2] M. Caccia, M. Bibuli, G. Bruzzone, G. Bruzzone, R. Bono, and E. Spirandelli, "Charlie, a testbed for usv research," IFAC Proceedings Volumes, vol. 42, no. 18, pp. 97 - 102, 2009, 8th IFAC Conference on Manoeuvring and Control of Marine Craft. [Online]. Available: http://www.sciencedirect.com/science/article/pii/S1474667016318778

[3] M. Dunbabin and A. Grinham, "Experimental evaluation of an autonomous surface vehicle for water quality and greenhouse gas emission monitoring," 062010 , pp. 5268 - 5274.

[4] C. R. Sonnenburg and C. A. Woolsey, "Modeling, identification, and control of an unmanned surface vehicle," Journal of Field Robotics, vol. 30, no. 3, pp. 371-398, 2013. [Online]. Available: https://onlinelibrary.wiley.com/doi/abs/10.1002/rob.21452

[5] D. Moreno, E. Besada, J. López, D. Chaos, J. Aranda, and J. Cruz, "Identificación de un modelo no lineal de un vehículo marino de superficie usando regresión simbólica," Actas de las Jornadas de Automática, pp. 850-855, 2015.

[6] S. Lack, E. Rentzow, and T. Jeinsch, "Experimental parameter identification for an open-frame rov: Comparison of towing tank tests and open water self-propelled tests," IFACPapersOnLine, vol. 52, no. 21, pp. 271 - 276, 2019, 12th IFAC Conference on Control Applications in Marine Systems, Robotics, and Vehicles CAMS 2019. [Online]. Available: http://www.sciencedirect.com/science/article/pii/S2405896319322049

[7] E. Morelli and V. Klein, Aircraft System Identification: Theory and Practice. Sunflyte Enterprises, 2016. [Online]. Available: https://books.google.com.pe/books?id=HsW-AQAACAAJ

[8] J. C. Cutipa-Luque and D. C. Donha, "Auv identification and robust control," IFAC Proceedings Volumes, vol. 44, no. 1, pp. 14735 - 14741, 2011, 18th IFAC World Congress. [Online]. Available: http://www.sciencedirect.com/science/article/pii/S1474667016459963

[9] S. Randeni, A. Forrest, R. Cossu, Z. Leong, D. Ranmuthugala, and V. Schmidt, "Parameter identification of a nonlinear model: replicating the motion response of an autonomous underwater vehicle for dynamic environments," Nonlinear Dynamics, 112017.

[10] C. Jian, Z. Jiayuan, X. Feng, Y. Jianchuan, Z. Zaojian, Y. Hao, $\mathrm{X}$. Tao, and Y. Luchun, "Parametric estimation of ship maneuvering motion with integral sample structure for identification," Applied Ocean Research, vol. 52, pp. 212 - 221, 2015. [Online]. Available: http://www.sciencedirect.com/science/article/pii/S0141118715000814

[11] M. Saccomani and L. D'Angió, "Examples of testing global identifiability with the daisy software," IFAC Proceedings Volumes, vol. 42, no. 10, pp. 48 - 53, 2009, 15th IFAC Symposium on System Identification. [Online]. Available: http://www.sciencedirect.com/science/article/pii/S1474667016386219

[12] N. Meshkat, C. E.-z. Kuo, and J. DiStefano III, "On finding and using identifiable parameter combinations in nonlinear dynamic systems biology models and combos: A novel web implementation," PLoS One, vol. 9, no. 10, p. e110261, 2014.

[13] A. F. Villaverde, A. Barreiro, and A. Papachristodoulou, "Strike-goldd user manual structural identifiability taken as extended-generalized observability with lie derivatives and decomposition," 2016.

[14] T. S. Ligon, F. Fröhlich, O. T. Chiş, J. R. Banga, E. Balsa-Canto, and J. Hasenauer, "GenSSI 2.0: multi-experiment structural identifiability analysis of SBML models," Bioinformatics, vol. 34, no. 8, pp. 1421-1423, 11 2017. [Online]. Available: https://doi.org/10.1093/bioinformatics/btx735

[15] B. A. Monroy-Ochoa and J. C. Cutipa-Luque, "Development of a low-cost unmanned surface vehicle for water quality monitoring," International Journal of Control and Automation, vol. 13, no. 4, pp. 1197-1207, Jul. 2020. 
[16] T. I. Fossen, Handbook of marine craft hydrodynamics and motion control. John Wiley \& Sons, 2011.

[17] J. N. Newman, Marine hydrodynamics. The MIT press (40th anniversary edition), 2018.

[18] L. Ljung, System Identification: Theory for the user, 2nd ed. Prentice
Hall, 1999.

[19] B. Maciel, L. Goes, E. Hemerly, and N. Neto, "Flight path reconstruction and parameter estimation using output-error method," Journal of Shock and Vibration, vol. 13, pp. 379-392, 2006. 